\title{
An Intramolecular Interaction between Src Homology 3 Domain and Guanylate Kinase-Like Domain Required for Channel Clustering by Postsynaptic Density-95/SAP90
}

\author{
Hyewon Shin, ${ }^{1}$ Yi-Ping Hsueh, ${ }^{2}$ Fu-Chia Yang, ${ }^{2}$ Eunjoon Kim, ${ }^{1}$ and Morgan Sheng ${ }^{2}$ \\ ${ }_{1}^{1}$ Department of Biological Sciences, Korea Advanced Institute of Science and Technology, Taejon 305-701, Korea, and \\ 2Howard Hughes Medical Institute and Department of Neurobiology, Massachusetts General Hospital and Harvard \\ Medical School, Boston, Massachusetts 02114
}

\begin{abstract}
Members of the postsynaptic density-95 (PSD-95)/SAP90 family of membrane-associated guanylate kinase (MAGUK) proteins function as multimodular scaffolds that organize proteinsignaling complexes at neuronal synapses. MAGUK proteins contain PDZ, Src homology $3(\mathrm{SH} 3)$, and guanylate kinase (GK)-like domains, all of which can function as sites for specific protein-protein interactions. We report here a direct proteinprotein interaction between the $\mathrm{SH} 3$ domain and the GK region in the PSD-95 family of MAGUKs. The SH3 domain of the PSD-95 family appears to have an atypical binding specificity, because the classical $\mathrm{SH} 3$ binding (-P-X-X-P-) motif is absent from the GK domain. Although SH3-GK binding can occur in
\end{abstract}

Synaptic transmission in neuronal synapses requires the proper organization of ion channels, neurotransmitter receptors, and signaling molecules in the synaptic junction. An emerging theme for the micro-organization of synapses is that functionally coupled proteins are placed in close proximity to each other via their interactions with scaffold proteins. The PSD-95 family of membrane-associated guanylate kinases (MAGUKs) plays such a role at postsynaptic sites of glutamatergic synapses by interacting with a variety of ion channels, receptors, cytoskeletal components, and intracellular signaling proteins (Sheng and Wyszynski, 1997; Ziff, 1997; Craven and Bredt, 1998; Kennedy, 1998; O’Brien et al., 1998).

The PSD-95 family of proteins (PSD-95/SAP90, SAP97/hdlg, chapsyn-110/PSD-93, and SAP102) share a common domain organization, consisting of three PDZ domains in their N-terminal half, a central Src homology 3 (SH3) domain, and a guanylate kinase (GK) domain at their $\mathrm{C}$ terminus. The PDZ domains of PSD-95 family proteins interact with the $\mathrm{C}$ terminal sequence motif (consensus $-\mathrm{X}-\mathrm{T} / \mathrm{S}-\mathrm{X}-\mathrm{V}$ ) found in a variety of membrane and intracellular proteins, including Shaker $\mathrm{K}^{+}$channels, NMDA receptors, and SynGAP (Kim et al., 1995, 1998; Kornau et al., 1995; Doyle et al., 1996; Chen et al., 1998). In addition, PSD-95

\footnotetext{
Received Dec. 2, 1999; revised Feb. 29, 2000; accepted March 9, 2000.

This work was supported by the Brain Science Research Program of Korea Ministry of Science and Technology, Korea Research Foundation Grants 98-019D00022 and 99-041-D00352, and the Korea Science and Engineering Foundation 1999 grant (E.K.). M.S. is Assistant Investigator of the Howard Hughes Medical Institute.

Correspondence should be addressed to Morgan Sheng, Howard Hughes Medical Institute (Wellman 423), Massachusetts General Hospital, 50 Blossom Street, Boston, MA 02114. E-mail: sheng@helix.mgh.harvard.edu.

Copyright (C) 2000 Society for Neuroscience $0270-6474 / 00 / 203580-08 \$ 15.00 / 0$
}

either an intramolecular or intermolecular manner, the intramolecular mode is preferred, possibly because of additional tertiary interactions available when the SH3 and GK domains are adjacent in the same polypeptide. Mutations disrupting the intramolecular SH3-GK interaction do not interfere with PSD-95 association with the $\mathrm{K}^{+}$channel Kv1.4 or with the GK domain-binding protein GKAP. The same mutations, however, inhibit the clustering of Kv1.4 by PSD-95, suggesting that the intramolecular SH3-GK interaction may modulate the clustering activity of PSD-95.

Key words: PDZ domain; ion channel clustering; postsynaptic density; Src tyrosine kinase; polyproline helix; disks large

binds to neuronal nitric oxide synthase (nNOS) through a PDZPDZ/ $\beta$-finger interaction (Brenman et al., 1996a; Hillier et al., 1999). In vitro and in vivo evidence suggests that these PDZmediated interactions are involved in the clustering of specific membrane proteins at synaptic sites (Kim et al., 1995, 1996; Thomas et al., 1997).

The GK domain of the PSD-95 family of MAGUKs interacts with the GKAP/SAPAP/DAP family of postsynaptic density proteins (Kim et al., 1997; Naisbitt et al., 1997; Takeuchi et al., 1997), BEGAIN (Deguchi et al., 1998), and MAP1A (Brenman et al., 1998). GKAP in turn interacts with a novel postsynaptic scaffold protein termed Shank (Lim et al., 1999; Naisbitt et al., 1999; Tu et al., 1999). Thus, the GK domain of PSD-95 can interact directly or indirectly with multiple postsynaptic proteins.

SH3 domains are found in many signaling and cytoskeletonassociated molecules, and are established as modular protein interaction domains with a binding specificity for certain prolinerich motifs (Pawson and Scott, 1997). Only one binding partner has been identified for the SH3 domain of PSD-95: the kainate receptor subunit KA2 (Garcia et al., 1998). The presence of an $\mathrm{SH} 3$ domain and its location adjacent to the GK domain are defining features of MAGUKs, implying an important function for this domain in the MAGUK superfamily. Consistent with this, a point mutation in the $\mathrm{SH} 3$ domain (m30 allele) of discs large-1 (dlg; a Drosophila homolog of PSD-95) causes a severe phenotype (Woods et al., 1996), including disruption of synaptic structure and neoplastic growth of the imaginal disks.

Here we report that the $\mathrm{SH} 3$ domain interacts in an intramolecular manner with the GK region of PSD-95 family proteins. This intramolecular interaction appears to be important for regulating the ion channel-clustering activity of PSD-95. 
A

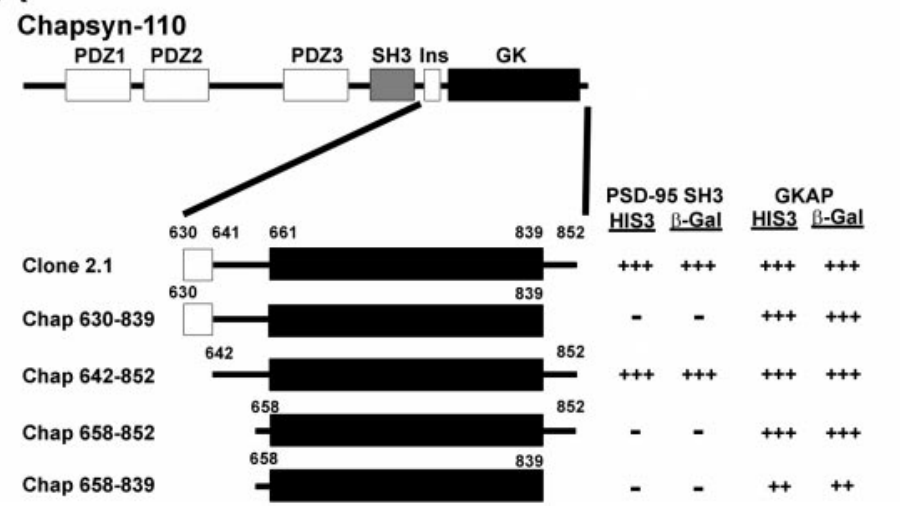

B

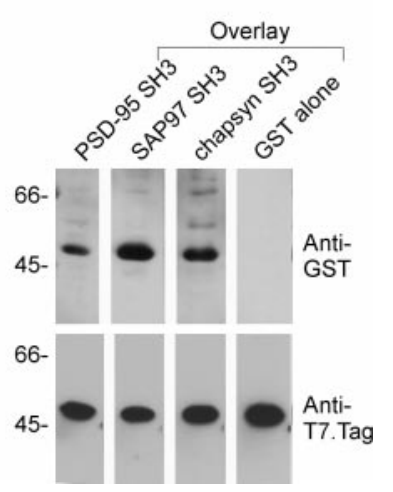

Figure 1. Interaction between $\mathrm{SH} 3$ domain and GK region in the yeast two-hybrid system and in filter-binding assays. $A$, Yeast two-hybrid screen using the PSD-95 SH3 domain as bait pulled out a cDNA fragment of chapsyn-110 (Clone 2.1). Clone 2.1 and further deletion variants are aligned under a schematic diagram of fulllength chapsyn-110. Interactions of these constructs with the PSD-95 SH3 domain or with GKAP are indicated, as measured by yeast two-hybrid assays based on induction of reporter genes HIS3 and $\beta$-galactosidase, as described in Kim et al. (1995). Numbers represent amino acid boundaries of domains or constructs. Ins, Alternatively spliced in-

sert (insertion 1) (Kim et al., 1996). B, Filter overlay assay showing direct binding of the SH3 domain and the GK region. Purified $\mathrm{H}_{6}$-fusion protein of the GK region of chapsyn-110 (clone 2.1) was separated by SDS-PAGE and transferred to a nitrocellulose membrane. Identical membranes were incubated with soluble GST-fusion proteins of the SH3 domains of PSD-95, SAP97, and chapsyn-110, or with control GST, as indicated. Bound GST-fusion proteins were visualized by anti-GST antibody (top panel). After stripping, the membrane was reprobed with anti-T7.Tag antibody to show the position and the relative abundance of the immobilized $\mathrm{H}_{6}$-fusion protein (bottom panel). Positions of molecular weight standards are indicated in kilodaltons.

\section{MATERIALS AND METHODS}

Yeast two-hybrid screening. Yeast two-hybrid screening was performed using the L40 yeast strain harboring HIS3 and $\beta$-gal as reporter genes, as described (Kim et al., 1995). The SH3 domain (aa 431-500) of PSD-95 was amplified by PCR and subcloned into pBHA (LexA binding domain) vector. This bait construct was used to screen an adult rat brain cDNA library constructed in pGAD10 (GAL4 activation domain vector; Clontech, Palo Alto, CA).

Analysis of the SH3-GK interaction by yeast two-hybrid assay. Deletion variants of clone 2.1 (chapsyn-110 aa 630-852) were made by PCR and subcloned into pGAD10. Interactions were tested in the yeast-twohybrid system using both HIS3 and $\beta$-gal as reporter genes. All constructs used in this study were confirmed by DNA sequencing.

The following yeast two-hybrid constructs contained the specified amino acids and were generated by PCR. pBHA constructs: SH3 domains: SAP97 (aa 580-652), chapsyn-110 (aa 539-608), dlg (aa 600680), ZO-1 (aa 583-801); GK regions: PSD-95 (aa 508-724), SAP97 (aа 656-911), chapsyn-110 (aa 630-852); SH3 + GK (S-G) domains: PSD-95 (aa 431-724), SAP97 (aa 580-911), chapsyn-110 (aa 522-852); SH3 + GK missing the last 13 amino acids (S-G $\Delta$ C): PSD-95 (aa 431-711), SAP97 (aa 580-898); S-G point mutations: PSD-95 S-G L460P, PSD-95 S-G W470A. pGAD10 constructs: SH3 domains: PSD-95 (aa 431-500), SAP97 (aa 581-648 of hdlg), chapsyn-110 (aa 539-608 of human chapsyn-110); GKr: PSD-95 (aa 508-724), SAP97 (aa 656-911), dlg (aa 728-960); GKr point mutations, PSD-95 P542A (proline-542 to alanine), PSD-95 P569A, PSD-95 P719A, PSD-95 P542A + P569A, PSD-95 P542A + P569A + P719A; SH3 + GK (S-G) domains: PSD-95 (aa 431-724), SAP97 (aa 580-911), chapsyn-110 (aa 522-852). pBHA-GKAP and pGAD10-GKAP have been described previously (Kim et al., 1997). Point mutations of the SH3 domain and GK region were made using QuickChange site-directed mutagenesis kit (Stratagene, La Jolla, CA).

Overlay filter binding assay. Overlay filter binding assay was performed as described (Kim et al., 1995). The chapsyn-110 cDNA (clone 2.1, an EcoRI fragment) was isolated from pGAD10 and subcloned in frame into $\mathrm{pRSETB}$ [hexahistidine $\left(\mathrm{H}_{6}\right)$-fusion protein vector; Invitrogen, San Diego, CA]. The SH3 domains of PSD-95 (aa 431-500), SAP97 (aa 581-648), and chapsyn-110 (aa 539-608) were amplified by PCR, digested, and subcloned in frame into pGEX4T-1 (GST fusion vector; Amersham Pharmacia Biotech). GST and $\mathrm{H}_{6}$-fusion proteins were purified using glutathione Sepharose 4B resin (Amersham Pharmacia Biotech) or Ni-NTA resin (Invitrogen). $\mathrm{H}_{6}$ fusion of the chapsyn-110 GK region was separated by $10 \%$ SDS-PAGE, transferred to a nitrocellulose membrane, and incubated with GST fusions of SH3 domains or GST fusion alone $(1 \mu \mathrm{g} / \mathrm{ml})$. Bound GST-fusion proteins were visualized by anti-GST antibody (Santa Cruz Biotechnology, Santa Cruz, CA) at a concentration of $1 \mu \mathrm{g} / \mathrm{ml}$.

COS cell transfection, clustering assay, pull-down assay, coimmunoprecipitation, and immunoblotting. Wild-type and mutant PSD-95 were ex- pressed using GW1 mammalian expression vector (British Biotechnology). GW1-PSD-95 missing the last 13 aa (PSD-95 $\Delta$ C) was made by amplifying PSD-95 (aa 1-711) by PCR and subcloning into GW1. GW1PSD-95s with point mutations (L460P and W470A) were made using QuickChange site-directed mutagenesis kit (Stratagene). N-PSD-95-GFP (termed N-GFP in Hsueh et al., 1997) has been described (Hsueh et al., 1997). GST-fusion protein constructs for pull-down assay were made by amplifying the SH3 domain (aa 408-509) and the SH3 + GK region (aa 431-724) of PSD-95 by PCR and subcloning into pGEX4T-1 (Amersham Pharmacia Biotech). GST-fusion protein containing the GKr (aa 630852 ) of chapsyn- 110 was made by subcloning the insert (clone 2.1) into pGEX4T-1. GST pull-down assays was performed as described (Naisbitt et al., 1999). Briefly, COS cells singly transfected with PSD-95 WT, L460P, or $\Delta \mathrm{C}$ were extracted with $1 \%$ Triton $\mathrm{X}-100$, followed by incubation with GST-fusion proteins and precipitation with glutathione Sepharose 4B resin (Amersham Pharmacia Biotech). Precipitates were analyzed by immunoblotting with PSD-95 antibody (K28/86). Coimmunoprecipitation and clustering assay were performed as described (Kim and Sheng, 1996; Kim et al., 1997). Membrane fractions of COS cells transfected with PSD-95 wild type and mutants were prepared as described (Hsueh and Sheng, 1999).

Kv1.4 (Sheng et al., 1992), PSD-95 (guinea pig, used for immunoprecipitation; Kim et al., 1995) and GKAP (Kim et al., 1997) antibodies have been described. Myc (9E10) and PSD-95 (K28/86, used for immunoblotting) monoclonal antibodies were purchased from Santa Cruz Biotechnology and Upstate Biotechnology (Lake Placid, NY), respectively.

\section{RESULTS \\ Interaction between the $\mathrm{SH} 3$ domain and the GK domain}

Using the SH3 domain of PSD-95 as yeast two-hybrid bait, we screened $\sim 0.5 \times 10^{6}$ clones of an adult rat brain cDNA library. A single interacting cDNA was isolated (clone 2.1), which encoded aa 630-852 of chapsyn-110/PSD-93, a close relative of PSD-95 that is also concentrated in postsynaptic densities (Brenman et al., 1996b; Kim et al., 1996). The clone 2.1 cDNA fragment begins 31 residues upstream of the GK domain of chapsyn-110 and extends through the very $\mathrm{C}$ terminus of the protein (residue 852 ) into the 3'UTR (Fig. 1A). Clone 2.1 did not interact with several control baits (including Kv1.4 C-terminal tail), thus its binding is specific for the PSD-95 SH3 domain. The interaction remained intact after reversing the bait and the prey in the yeast two-hybrid system (data not shown).

$\mathrm{N}$-terminal and C-terminal deletion constructs of clone 2.1 were tested for interaction with the PSD-95 SH3 domain in the 
Figure 2. Intragenic and intergenic interactions between SH3 domains and GK regions of PSD-95 family of proteins. $A$, SH3 domains from PSD-95, SAP97, chapsyn-110, and Dlg were tested for interaction with GK regions from PSD-95, SAP97, chapsyn-110, and Dlg in the yeast two-hybrid system (as in Fig. 1A). SH3 domains were fused to the LexA DNA-binding domain (pBHA vector), and GK regions were fused to the GAL4 activation domain (pGAD10 vector). Empty pGAD10 vector was negative control. Blank areas were not tested. $B$, Interaction between $\mathrm{SH} 3$ domain and proline mutants of PSD-95 GK region. GK region constructs containing single or multiple proline-toalanine mutations in the indicated sequences were tested for binding to the SH3 domain of PSD-95, or to GKAP. $W T$, Wild type. $C$, Interaction between the GK region of PSD-95 family proteins and mutants of the SH3 domain of PSD-95. Substitution mutants (L460P and W470A) of the SH3 domain of PSD-95 do not interact with GK regions from PSD95, SAP97, and chapsyn-110.

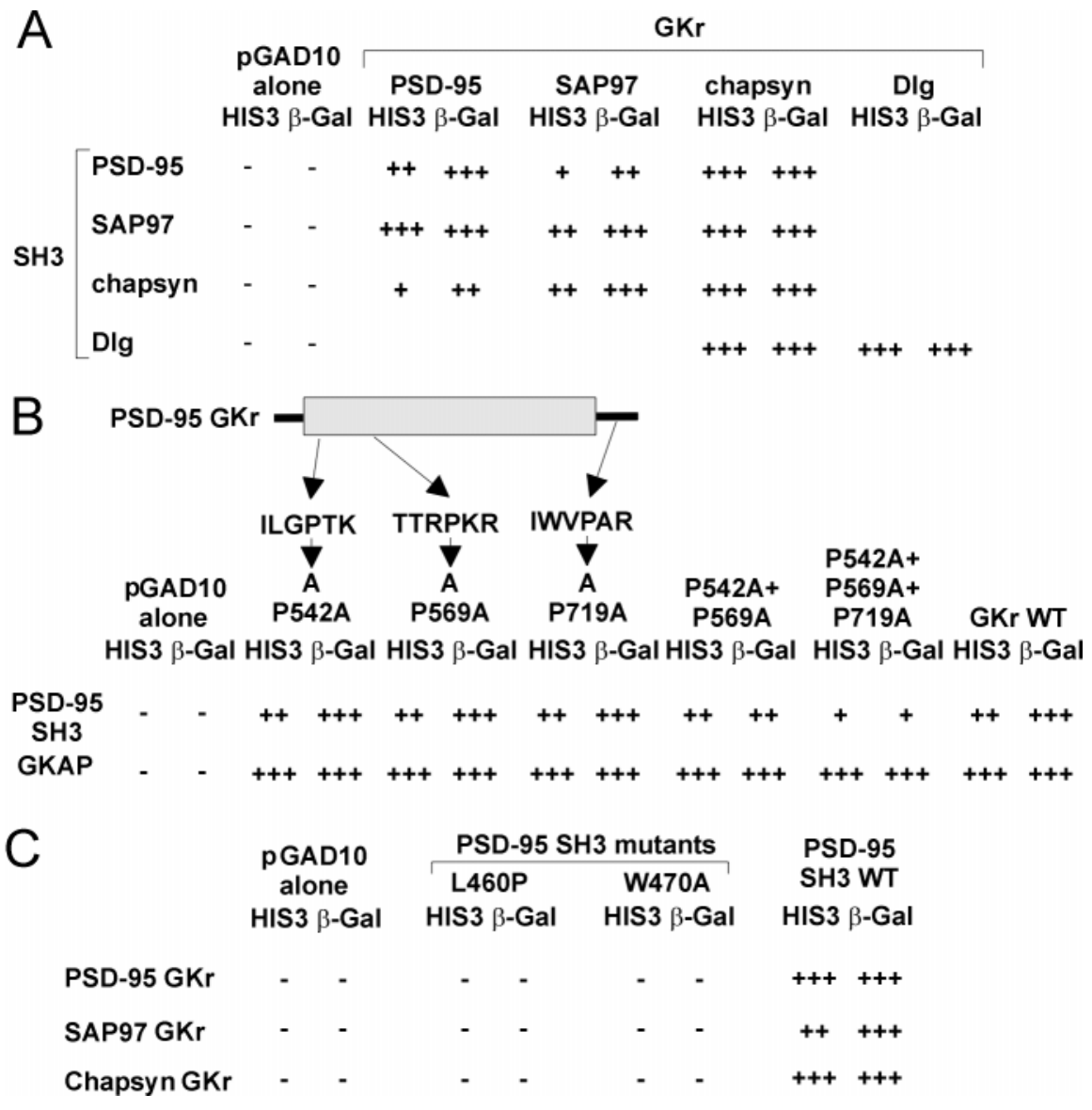

tem. As shown in Figure $2 A$, the PSD-95 SH3 domain can interact not only with the PSD-95 GK region but also with the GK regions from SAP97 and chapsyn-110. Similar results were obtained with the SH3 domains from SAP97 and chapsyn-110 (Fig. $2 A)$. In addition, the $\mathrm{SH} 3$ domain of the Drosophila homolog Dlg was able to bind to the GK region of Dlg and to the GK region of chapsyn-110 (Fig. 2A). These results are not surprising given that within the PSD-95/Dlg subfamily of MAGUKs there is high conservation in the SH3 and GK domains (65-75\% amino acid sequence identity). No significant interaction was detectable between the SH3 domain of SAP97 and the GK region of ZO-1, a distantly related MAGUK (data not shown). Thus SH3 domains from the PSD-95 family of proteins appear to interact specifically with GK regions from within the PSD-95 family, but not with GK regions from more distantly related MAGUKs. In fact, there is only $\sim 30 \%$ sequence identity between the GK domains of PSD-95 and ZO-1.

SH3 domains typically interact with proline-rich motifs. The GK region of PSD-95 has a total of 10 prolines, none of which conforms to the classical PXXP consensus for SH3 recognition. However, three of these prolines lie in a class II polyproline helix-like sequence (XXXPXR/K) that has been shown to bind some SH3 domains (Sicheri et al., 1997; Xu et al., 1997). The most C-terminal of these class II-like sequences (IWVPAR, Fig. 2B) is located in the middle of the 13 residue tail that follows the GK domain and is intriguing because truncation of the $\mathrm{C}$ terminus of Dlg results in a tumorigenic phenotype in flies (Woods and Bryant, 1991). We tested whether these three class II prolinecontaining sequences are involved in binding to the $\mathrm{SH} 3$ domain by mutating the prolines to alanines, individually and in combiintragenic and intergenic manner using the yeast two-hybrid sys(he PSD-95 family (chapsyn-110/PSD-93) can bind to its own $\mathrm{SH} 3$ domain as well as to $\mathrm{SH} 3$ domains from PSD-95 and SAP97. We tested systematically whether SH3 and GK regions from within the PSD-95 family can interact in an 


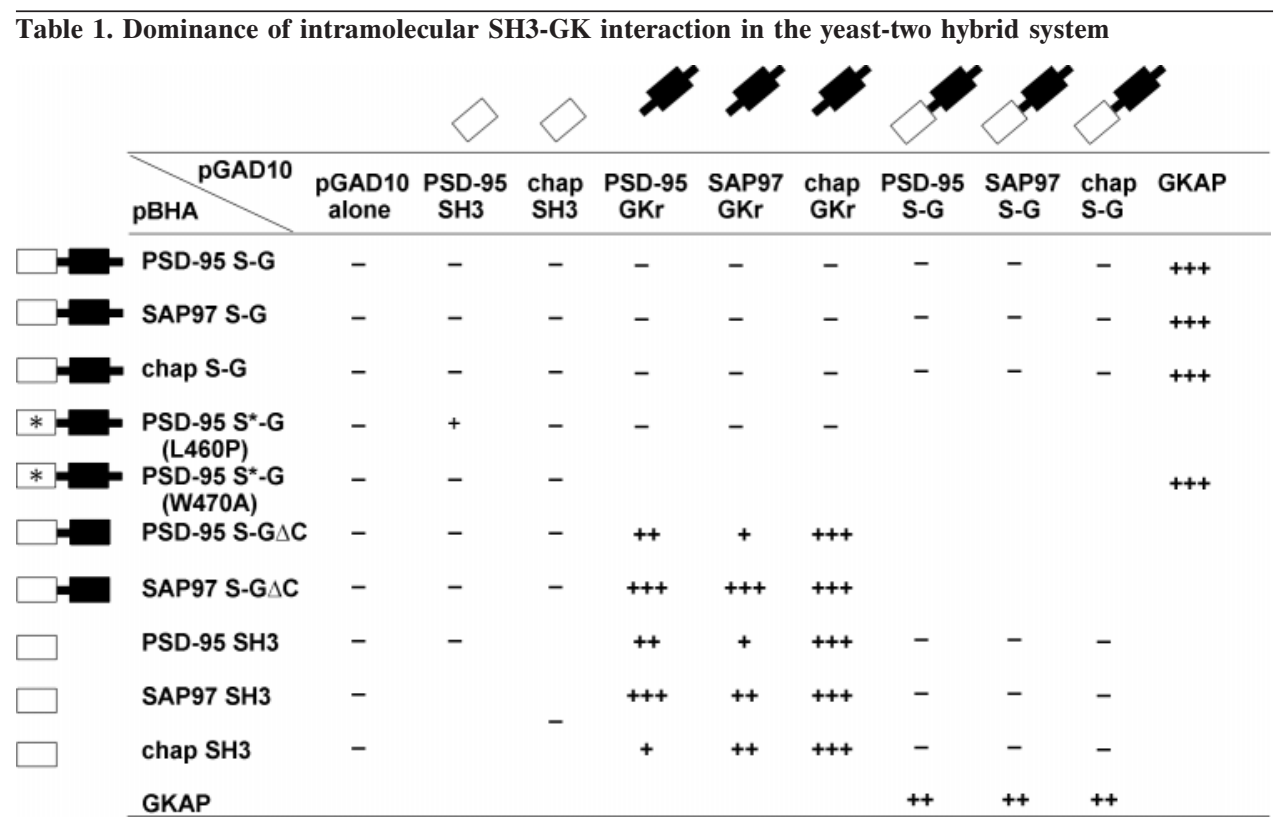

The contiguous SH3 + GK domains (S-G) from PSD-95, SAP97, and chapsyn-110 do not interact with exogenous SH3 domains, GK domains, or S-G domains while interacting with GKAP. The S-G construct of PSD-95, with a L460P mutation in the SH3 domain (S*-G(L460P)) can interact with the SH3 domain of PSD-95, whereas S $^{*}$ G(W470A) does not interact with the SH3 domains. Unlike S-G constructs, the $\mathrm{S}-\mathrm{G} \Delta \mathrm{C}$ constructs of PSD-95 and SAP97 (missing the C-terminal 13 amino acids) can interact with GK regions. In the reverse orientation of the yeast two-hybrid, SH3 domains from PSD-95, SAP97, and chapsyn-110 do not interact with the S-G constructs from PSD-95, SAP97, and chapsyn-110. Activation of reporter genes was semiquantified as in Figure 1 ; only results of $\beta$-galactosidase assays are shown. Results of HIS3 activation agree with those of $\beta$-galactosidase activation (data not shown). The constructs depicted in the first column were subcloned into pBHA (LexA DNA binding domain) vector, and the constructs in the first row were subcloned into pGAD10 (GAL4 activation domain) vector. Blank areas were not tested.

nation. None of the individual proline mutations affected the SH3-GKr interaction or the GKAP-GKr interaction in yeast two-hybrid assays (Fig. 2B). SH3 binding was retained even with the double mutation (P542A/P569A) and the triple mutation (P542A/P569A/P719A) in the GK region, although the strength of interaction in the yeast two-hybrid system became weaker with increasing number of substitutions (Fig. 2B). These data indicate that the class II-like proline-containing sequences are not essential for $\mathrm{SH} 3$ binding.

The m30 point mutation in the $\mathrm{SH} 3$ domain of $d l g$ causes a severe phenotype in Drosophila (Woods et al., 1996). An equivalent point mutation (leucine to proline) in residue 460 of PSD-95 (L460P) disrupted the interaction between the SH3 domain of PSD-95 and the GK region of PSD-95 family proteins (Fig. 2C). These data indicate that leucine-460 (L460) in the SH3 domain is important for the $\mathrm{SH} 3-\mathrm{GKr}$ interaction and raise the possibility that the functional impairment of the $d l g \mathrm{~m} 30$ allele could result at least in part from the loss of $\mathrm{SH} 3-\mathrm{GKr}$ interaction.

It was recently reported that the SH3 domain of PSD-95/SAP90 interacts with the $\mathrm{C}$-terminal proline-rich region of kainate receptor subunit KA2, which contains several PXXP motifs (Garcia et al., 1998). Mutating amino acid 470 in the SH3 domain of PSD-95 from tryptophan to alanine (W470A), a substitution known to prevent the interaction between $\mathrm{SH} 3$ domains and proline-rich motifs, disrupted PSD-95-KA2 binding (Garcia et al., 1998). We introduced the W470A mutation into the SH3 domain of PSD-95 and found that it abolished SH3 interaction with the GK regions of PSD-95 family proteins in yeast two-hybrid assays (Fig. 2C).

\section{Intramolecular interaction between the SH3 domain and the GK region}

The yeast two-hybrid and in vitro binding data presented above demonstrate an intermolecular interaction between the $\mathrm{SH} 3$ domain and the GK region. To test whether the interaction between the $\mathrm{SH} 3$ domain and the GK region can also occur in an intramolecular (cis) mode, we made bait constructs of PSD-95, SAP97, and chapsyn-110 in which the SH3 domain and GK region are covalently linked together in their native contiguous state ("S-G" constructs). These $\mathrm{S}-\mathrm{G}$ constructs were tested for interaction in trans with GAL4 activation domain fusions of SH3 or GK domains in the yeast two-hybrid system. The $\mathrm{S}-\mathrm{G}$ constructs did not interact with either SH3 domains or GK regions (Table 1), suggesting that the intramolecular (cis) interaction is preferred over the intermolecular (trans) interaction. Note however that all the $\mathrm{S}-\mathrm{G}$ constructs were able to interact with GKAP in parallel assays (Table 1, last column), indicating that these $S-G$ constructs were functional in the two-hybrid system and further emphasizing the different mode of binding of GKAP to the GK domain. In addition to providing a positive control for this experiment, these results indicate that the intramolecular interaction between the SH3 domain and the GK region does not interfere with the binding of GKAP to the GK domain (Table 1, last column).

The C-terminal 13 amino acid tail following the GK domain is required for $\mathrm{SH} 3$ binding to the $\mathrm{GK}$ region (Fig. $1 A$ ). Interestingly, deleting the last 13 amino acids from the $\mathrm{S}-\mathrm{G}$ constructs of PSD-95 or SAP97 (giving S-G $\Delta$ C constructs) caused them to regain their ability to interact in trans with GK regions (Table 1, middle two rows). The most likely explanation for this result is 
that deleting the $\mathrm{C}$-terminal 13 aa disrupted the intramolecular interaction within the $\mathrm{S}-\mathrm{G}$ bait construct, thereby liberating the $\mathrm{SH} 3$ domain to bind in trans to the GK region. The $\mathrm{S}-\mathrm{G} \Delta \mathrm{C}$ constructs, however, did not interact in trans with $\mathrm{SH} 3$ domains (Table 1), presumably because the 13 amino acid deletion removed the C-terminal site required for SH3 interaction. Similarly, an S-G construct of PSD-95 with a point mutation (L460P) in the SH3 domain ( $\left.\mathrm{S}^{*}-\mathrm{G}(\mathrm{L} 460 \mathrm{P})\right)$ interacted in trans with the SH3 domain of PSD-95, but not with the GK region (Table 1, row 4). This is the expected result if the L460P mutation disrupted the intramolecular interaction between $\mathrm{SH} 3$ domain and GK region, liberating the intact GK region for binding in trans to a wild-type SH3 domain. The binding between $\mathrm{S}^{*}-\mathrm{G}(\mathrm{L} 460 \mathrm{P})$ and PSD-95 SH3 domain appeared weaker than between the isolated GK region and SH3 domain (Table 1, Fig. 2), for unknown reasons. We could not detect an interaction between PSD-95 $\mathrm{S}^{*}$ G(L460P) and the SH3 domain of chapsyn-110, perhaps related to the fact that the interaction between the GKr of PSD-95 and the SH3 domain of chapsyn-110 was already weaker than with the PSD-95 SH3 domain (Fig. 2A). Finally, because an S-G construct cannot interact in trans with either a $\mathrm{SH} 3$ domain or a $\mathrm{GK}$ region, it was not surprising that no interaction was detectable between two separate $\mathrm{S}-\mathrm{G}$ constructs (Table 1).

We have shown above that the W470A mutation in the SH3 domain of PSD-95 disrupts the intermolecular interaction between $\mathrm{SH} 3$ domain and GK regions in the yeast two-hybrid assay (Fig. 2C). We further tested if the same mutation also disrupts the intramolecular interaction in the yeast two-hybrid system. Interestingly, a S-G construct of PSD-95 containing the W470A mutation ( $\mathrm{S}^{*}-\mathrm{G}(\mathrm{W} 470 \mathrm{~A})$ ) showed no interaction with $\mathrm{SH} 3$ domains from PSD-95 or chapsyn-110 while interacting robustly with GKAP (Table 1, row 5). This finding suggests that the W470A mutation did not disrupt the intramolecular interaction between $\mathrm{SH} 3$ and GKr in the S-G construct.

In the above yeast two-hybrid experiments, dimerization of the LexA DNA binding domain could lead to dimerization of the S-G constructs. Trans interactions between SH3 domains and GK regions located in separate but dimerized bait molecules could explain the behavior of $\mathrm{S}-\mathrm{G}$ constructs rather than intramolecular SH3-GK interactions. To test this possibility, we asked whether the PSD-95 SH3 domain fused to LexA can interact with the SH3-GK $(\mathrm{S}-\mathrm{G})$ region of PSD-95, SAP97 and chapsyn-110 fused to the GAL4 activation domain (which does not dimerize). Negative results were also obtained from this experiment (Table 1), further supporting the idea that the cis interaction within $\mathrm{S}-\mathrm{G}$ constructs is preferred over the trans interaction with $\mathrm{SH} 3$ domains. Moreover, a LexA bait construct containing the PSD-95 GK region was able to interact with the PSD-95 SH3 domain in pGAD10, but not with the PSD-95 S-G construct (data not shown). Thus, the intramolecular interaction of $\mathrm{S}-\mathrm{G}$ constructs also seems to prevent interaction in trans with isolated GK regions.

We next tested whether the intramolecular SH3-GKr interaction was dominant in the context of PSD-95 protein expressed in mammalian cells. When expressed in COS cells, the SH3 domain mutant PSD-95(L460P), but not wild-type PSD-95 or PSD-95 C, could be "pulled down" by GST-fusion protein of the SH3 domain of PSD-95 (Fig. 3A, lanes 4-6). These results are explained if the L460P mutation disrupts intramolecular SH3-GKr interaction, thereby freeing the intact GK region to bind to GST-SH3. Similarly, PSD-95 $\Delta$ C, but not wild-type PSD-95 or PSD95(L460P), was pulled down by GST-fusion protein containing the GK region of PSD-95 (Fig. 3A, lanes 13-15). Further supporting an intramolecular SH3-GKr interaction, GST-fusion protein of the combined $\mathrm{SH} 3$ and $\mathrm{GK}$ regions $(\mathrm{S}-\mathrm{G})$ could not pull-down any PSD-95 protein expressed in COS cells, whether wild-type or L460P (Fig. 3A, lanes 7-12). The same GST-SH3-GK-fusion protein, however, pulled down GKAP proteins expressed in COS cells (data not shown).

\section{Disruption of the SH3-GKr interaction impairs PSD-95 clustering activity}

We tested if the SH3-GKr interaction had any functional consequences on protein-protein interactions or ion channel-clustering activity of PSD-95 in COS cells. In COS cells doubly transfected with $\mathrm{K}^{+}$channel Kv1.4 and PSD-95 or PSD-95(L460P) or PSD$95 \Delta \mathrm{C}$, the amount of Kv1.4 coimmunoprecipitated with PSD-95 antibodies was not significantly different between wild-type and mutant PSD-95 proteins (Fig. 3B, lanes 4-6). Similar results were obtained with GKAP (Fig. 3C, lanes 4-6). Thus, disrupting the intramolecular SH3-GKr interaction by mutating the $\mathrm{SH}$ domain (L460P) or the GK region $(\Delta \mathrm{C})$ had no obvious effect on the association of PSD-95 with its binding partners Kv1.4 or GKAP in mammalian cells. In control experiments, Kv1.4 and GKAP were not immunoprecipitated by PSD-95 antibodies in the absence of coexpression of wild-type or mutant PSD-95 (data not shown).

COS cells doubly transfected with Kv1.4 and wild-type PSD-95 formed large surface-associated coclusters of Kv1.4 and PSD-95 (Fig. 3D, top panels; $\sim 65 \%$ of cotransfected cells showed such clusters), as shown previously (Kim et al., 1995; Kim and Sheng, 1996). Interestingly, COS cells doubly transfected with Kv1.4 and PSD-95(L460P) or with Kv1.4 and PSD-95 $\Delta$ C did not form any typical clusters. Instead, Kv1.4 and these mutant PSD-95 proteins were diffusely distributed throughout the cells, or were sometimes colocalized in perinuclear clusters (Fig. 3D), the latter reminiscent of SAP97 aggregates trapped in the endoplasmic reticulum (Tiffany et al., 2000). Because both the L460P and $\Delta \mathrm{C}$ mutations impair SH3-GKr interaction, but not Kv1.4 binding by PSD-95, these results suggest that the $\mathrm{S}-\mathrm{G}$ intramolecular interaction is important for the ion channel-clustering activity of PSD-95. Finally, we tested the clustering behavior of PSD-95 containing the W470A mutation. This mutation, which disrupts the intermolecular but not the intramolecular SH3-GKr interaction in yeast two-hybrid assays (Table 1), also did not affect the ability of PSD-95 to form normal clusters with Kv1.4 (Fig. 3D, bottom panels). Thus, channel-clustering ability is correlated with intramolecular SH3-GK interaction.

To probe the mechanism underlying the loss of channelclustering activity of PSD-95 mutants L460P and $\Delta \mathrm{C}$, we tested these mutant proteins for multimerization and membrane association, which are important steps in the process of Kv1.4 clustering by PSD-95 (Hsueh et al., 1997; Topinka and Bredt, 1998; Hsueh and Sheng, 1999). In a COS cell coimmunoprecipitation assay, PSD-95 mutants L460P and $\Delta \mathrm{C}$ were able to associate with a PSD-95-GFP-fusion protein containing the N-terminal multimerization domain of PSD-95 (N-PSD-95-GFP; Fig. 4A, lanes 7, 8). The coimmunoprecipitation of these PSD-95 mutants (L460P and $\triangle \mathrm{C}$ ) with N-PSD-95-GFP was actually better than wild-type PSD-95 (Fig. 4A, lane 6). It is notable that PSD-95 with the W470A mutation (which has an intact intramolecular SH3-GKr interaction) showed association with N-PSD-95-GFP comparable to wild-type PSD-95 (Fig. 4A, lane 9). As control, the PSD-95 guinea pig antibody used for immunoprecipitation did not pre- 


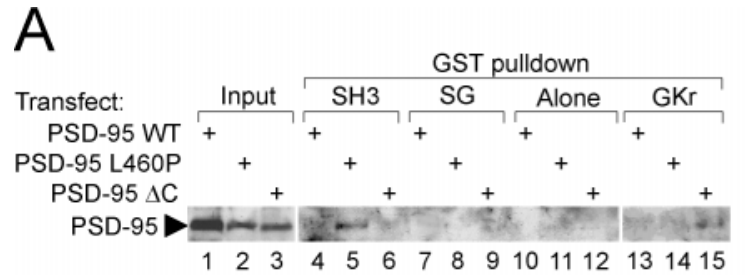

B

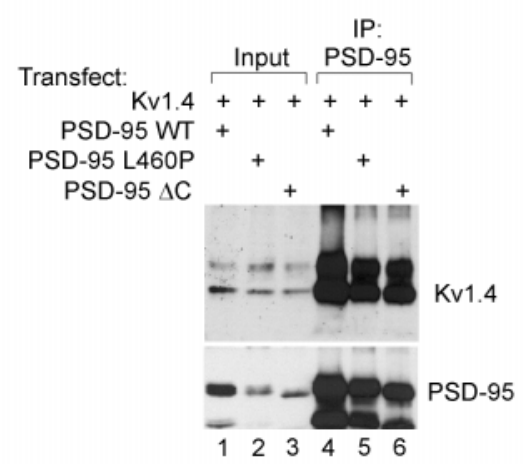

C
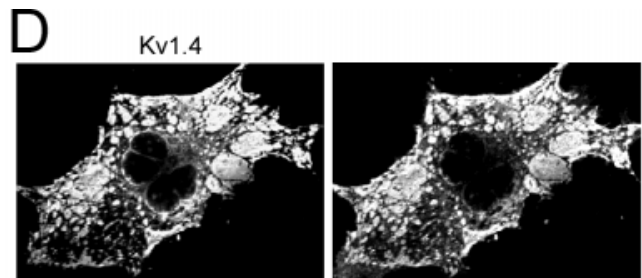

PSD-95
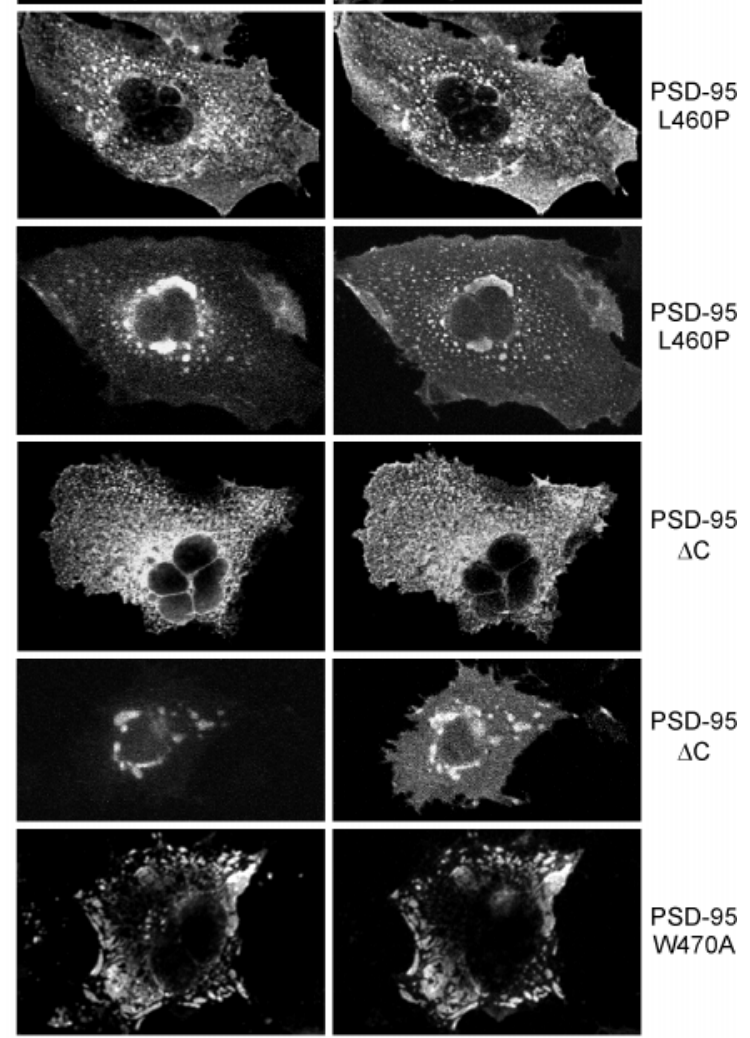

Figure 3. PSD-95 mutants defective in intramolecular SH3-GK interaction still bind to Kv1.4 and GKAP, but cannot cluster Kv1.4. A, GST pull-down assay confirming dominance of the intramolecular SH3-GK interaction in mammalian cells. Wild-type and mutant (L460P and $\Delta$ C) PSD-95 proteins expressed in COS cells were incubated with GST-fusion proteins of the PSD-95 SH3 domain, GK region, or combined SH3-GK (SG) regions, or with GST alone. Precipitates were immunoblotted with PSD-95 antibody. Input, 10\% of COS cell extract used in pull-down assay. $B$, Coimmunoprecipitation of Kv1.4 with wild-type and mutant PSD-95 proteins. COS cells doubly transfected with Kv1.4 and wild-type or mutant (L460P and $\Delta$ C) PSD-95 were immunoprecipitated with PSD-95 antibody, and the precipitates immunoblotted for Kv1.4 and PSD-95. Input, 5\% of the extract used in the immunoprecipitation. $C$, Coimmunoprecipitation of GKAP with wild-type and mutant PSD-95 proteins. COS cells were doubly transfected with GKAP and wild-type and mutant PSD-95, and immunoprecipitated with PSD-95 antibody, as in $B$, but the precipitates were immunoblotted for GKAP and PSD-95. D, Kv1.4 clustering by wild-type and mutant PSD-95 proteins. COS cells were doubly transfected with Kv1.4 and wild-type and mutant forms of PSD-95, as indicated. Distribution of Kv1.4 and PSD-95 was examined by double label immunofluorescence with Kv1.4 (left) and PSD-95 antibodies (right). Typical plaque-like coclusters formed between Kv1.4 and wild-type PSD-95 (top panels), but were not seen with PSD-95 mutants L460P and $\Delta \mathrm{C}$ (middle panels). Instead, Kv1.4 and PSD-95 mutants were mainly diff use; colocalization of these proteins occurred only in fine scattered puncta or in large perinucelar clusters. PSD-95(W470A) mutant, however, formed wild-type-looking clusters with Kv1.4 (bottom panels).

cipitate N-PSD-95-GFP in the absence of full-length PSD-95 (Fig. 4A, lane 10).

In membrane fractionation experiments, L460P and $\Delta \mathrm{C}$ mutants of PSD-95 expressed in COS cells showed membrane association and membrane extractability properties that were similar to wild-type PSD-95 (Fig. 4B). Taken together, these results indicate that the loss of clustering activity in PSD-95 L460P and $\Delta \mathrm{C}$ cannot be attributed simply to loss of multimerization or membrane association of these PSD-95 mutants.

\section{DISCUSSION}

\section{Atypical binding specificity of the SH3 domains of PSD-95 family}

SH3 domains generally interact with short peptides that form a polyproline helix of two types: class I (consensus RXLPPdPXX, where $\mathrm{d}$ is $\mathrm{L}$ for Src and $\mathrm{R}$ for PI3 kinase) and class II (XXXPPLPXR for both Src and PI3 kinase) (Cohen et al., 1995). Within the PSD-95 family, a search failed to yield any PXXP sequence (the classical minimal consensus for SH3 interaction) in the GK region. Furthermore, mutation of the three class II-like XXX$\mathrm{PXR} / \mathrm{K}$ sequences in the GK region did not prevent SH3-GKr interaction. Thus, the SH3 domains of PSD-95 MAGUKs seem to have an atypical ligand binding specificity, distinct from classical SH3 domains. Indeed, sequence comparisons of the SH3 domains from the PSD-95 family and other MAGUKs (p55, CASK, Dlg, and ZO-1) with the "classical" SH3 domains from c-Src, c-Abl, and Grb2 reveal that some of the critical residues for PXXP recognition are not conserved in the $\mathrm{SH} 3$ domains of MAGUK proteins. For instance, among the important residues in c-Src (Y90, Y92, D99, W118, Y131, P133, and Y136), D99 and 


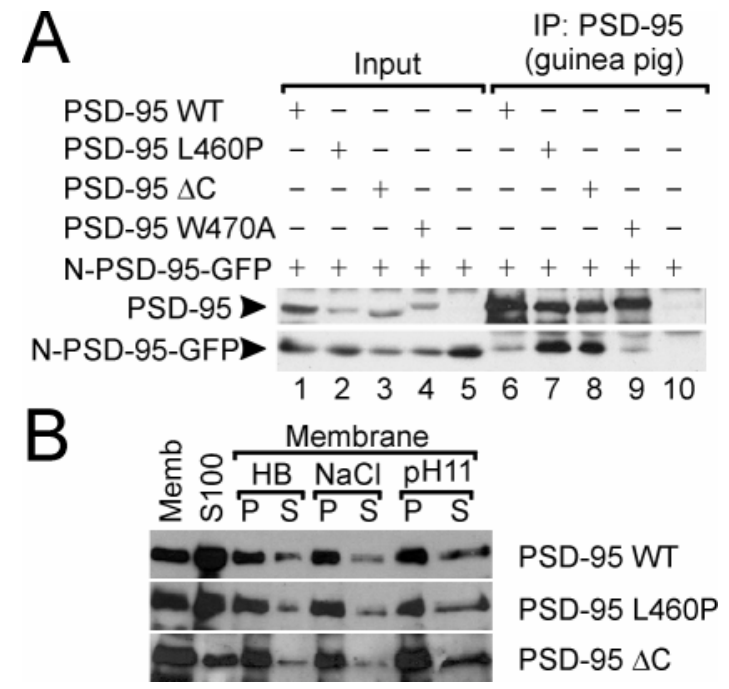

Figure 4. PSD-95 mutants defective in intramolecular SH3-GK interaction show normal multimerization and membrane association. $A, \mathrm{COS}$ cell lysates doubly transfected with N-PSD-95-GFP (containing the $\mathrm{N}$-terminal multimerization domain of PSD-95) and wild-type or mutant PSD-95, as indicated, were immunoprecipitated with PSD-95 (guinea pig) antibody and probed with PSD-95 (top panel) and myc antibodies (for N-PSD-95-GFP, bottom panel). B, COS cells were transfected with wildtype or mutant (L460P or $\Delta \mathrm{C}$ ) PSD-95. Membrane (Memb) and cytosolic S100 fractions (S100) were prepared as described in Materials and Methods. The membranes were further extracted with isotonic homogenate buffer $(\mathrm{HB}), 1 \mathrm{M}$ sodium chloride $(\mathrm{NaCl})$, or alkaline sodium carbonate buffer $(p H 11)$ to yield pellet $(P)$ and soluble fractions $(S)$.

Y136 are substituted by $\mathrm{G} / \mathrm{A}$ and $\mathrm{E} / \mathrm{R} / \mathrm{K} / \mathrm{N}$ respectively, in the various MAGUKs. The lack of conservation of the D99 and Y136 equivalent residues in the SH3 domains of PSD-95 family MAGUKs could account for their binding of non-PXXP target sequences. Nevertheless, the inhibitory effect of the W470A mutation (equivalent to W118 of Src SH3 domain) on intermolecular SH3 binding to the GK region is consistent with the idea that the PSD-95 SH3 domain uses the equivalent peptide binding surface as used by the Src SH3 domain.

Given that interpretation, it is significant that the same $\mathrm{SH} 3$ mutation (W470A) that abolishes intermolecular SH3-GKr association does not prevent intramolecular SH3-GKr interaction. We interpret this data as indicating that the intramolecular (cis) interaction between the $\mathrm{SH} 3$ domain and the GK region involves specific contacts in addition to those involving tryptophan-470. These additional interactions could be facilitated by the linkage of SH3 and GK domains close to each other in the same polypeptide and would explain why the intramolecular interaction is favored over the intermolecular interaction. The additional interactions in the cis configuration may involve binding surfaces distinct from the conventional peptide-binding groove the $\mathrm{SH} 3$ domain of PSD-95. Such intramolecular tertiary interactions involving surfaces outside the peptide-binding groove have been reported for the SH3 domain in Src family tyrosine kinases (Xu et al., 1997) (see below).

\section{Potential functions of intramolecular SH3-GK region interaction}

Intramolecular interactions involving the SH3 domain are well established in the Src family of nonreceptor tyrosine kinases and are important for keeping the kinase in an catalytically inactive state. The SH3 domain of Src and Hck interacts with a type II polyproline segment in the Src homology 2 (SH2) kinase linker region and in addition directly contacts the kinase domain through parts of the SH3 domain distinct from the core ligandbinding surface (Moarefi et al., 1997; Pawson and Scott, 1997; Xu et al., 1997). Our results reveal intriguing parallels between the intramolecular SH3 interaction in PSD-95 MAGUKs and that in Src-like kinases. The SH3 domain in both proteins lies N-terminal to the tyrosine or guanylate kinase domains, and in both cases, intramolecular SH3 interactions appears to involve regions of the protein within and flanking the kinase domains. Truncation of the C-terminal tail of Dlg results in a tumorigenic phenotype, reminiscent of Src activation by loss of its $\mathrm{C}$ terminus. The analogy with Src family kinases prompts us to speculate that an intramolecular interaction between the $\mathrm{SH} 3$ domain and the GK region may be involved in regulating the activity of the $\mathrm{SH} 3$ or GK domain in MAGUK proteins. Currently, no enzymatic activity is attributed to the GK domain of PSD-95 family proteins (Kuhlendahl et al., 1998). However, the "closed" (cis-interacting) conformation of the SH3-GK region might prevent binding of ligands to the $\mathrm{SH} 3$ domain or to the GK domain. Binding of high-affinity ligands to the SH3 domain of PSD-95 could "unfold" the SH3-GK region and allow access of proteins to the GK domain. Potential ligands include kainate receptor KA2 that binds to the SH3 domain of PSD-95 (Garcia et al., 1998) or calmodulin that binds to the region around the C-terminal boundary of the SH3 domain of the PSD-95 family member SAP102 (Masuko et al., 1999). So far, we have found no evidence that the intramolecular SH3-GK interaction prevents binding of GKAP to the GK domain, but other GK-binding proteins like BEGAIN remain to be examined.

In this study, the major functional effect of disrupting the intramolecular SH3-GK interaction was to impair the normal ability of PSD-95 to cluster the $\mathrm{K}^{+}$channel Kv1.4. This effect is relatively specific because the same mutations in the PSD-95 protein (L460P and $\Delta \mathrm{C}$ ) had no effect on Kv1.4 binding or membrane association of PSD-95; nor did they disrupt head-tohead multimerization of PSD-95. Because, if anything, the L460P and $\Delta \mathrm{C}$ mutations would enhance cross-linking of PSD-95 by promoting intermolecular (trans) SH3-GK association, their deleterious effects specifically on channel clustering carries more significance. We therefore propose that the SH3-GK interaction has some intramolecular regulatory function, rather than an intermolecular cross-linking function, that is important for the channel-clustering property of PSD-95. The precise mechanism by which the intramolecular SH3-GK interaction is involved in channel clustering by PSD-95 remains to be resolved. Our observation that $\mathrm{L} 460 \mathrm{P}$ and $\Delta \mathrm{C}$ mutants often form perinuclear clusters with Kv1.4 raises the additional possibility that an intact SH3GKr interaction is required for proper intracellular trafficking of PSD-95 (Arnold and Clapham, 1999; Craven et al., 1999; ElHusseini et al., 2000; Tiffany et al., 2000). Whatever the precise mechanism, this study raises the interesting idea that regulation of the intramolecular SH3-GK interaction (e.g., by binding of GK or SH3 ligands, or by phosphorylation) could control the targeting and clustering activity of PSD-95 at postsynaptic sites. Interestingly, $\mathrm{SH} 3$ and $\mathrm{C}$-terminal mutations equivalent to those that abolish channel clustering by PSD-95 have been isolated as severe mutant alleles of discs large in Drosophila (Woods and Bryant, 1991; Lahey et al., 1994). Further characterization of the protein interactions mediated by the $\mathrm{SH} 3$ and GK domains should increase our understanding of the function and regulation of PSD-95 and other MAGUKs at cell junctions. 
Note added in revision. McGee and Bredt (1999) have also reported an intramolecular interaction between the $\mathrm{SH} 3$ domain and the GK region of PSD-95. These investigators found that a W470F mutation did not abolish intermolecular SH3-GK interaction.

\section{REFERENCES}

Arnold DB, Clapham DE (1999) Molecular determinants for subcellular localization of PSD-95 with an interacting $\mathrm{K}+$ channel. Neuron 23:149-157.

Brenman JE, Chao DS, Gee SH, McGee AW, Craven SE, Santillano DR, Wu Z, Huang F, Xia H, Peters MF, Froehner SC, Bredt DS (1996a) Interaction of nitric oxide synthase with the postsynaptic density protein PSD-95 and alpha1-syntrophin mediated by PDZ domains. Cell 84:757-767.

Brenman JE, Christopherson KS, Craven SE, McGee AW, Bredt DS (1996b) Cloning and characterization of postsynaptic density 93, a nitric oxide synthase interacting protein. J Neurosci 16:7407-7415.

Brenman JE, Topinka JR, Cooper EC, McGee AW, Rosen J, Milroy T, Ralston HJ, Bredt DS (1998) Localization of postsynaptic density-93 to dendritic microtubules and interaction with microtubule-associated protein 1A. J Neurosci 18:8805-8813.

Chen HJ, Rojas-Soto M, Oguni A, Kennedy MB (1998) A synaptic Ras-GTPase activating protein (p135 SynGAP) inhibited by CaM kinase II. Neuron 20:895-904.

Cohen GB, Ren R, Baltimore D (1995) Modular binding domains in signal transduction proteins. Cell 80:237-248.

Craven SE, Bredt DS (1998) PDZ proteins organize synaptic signaling pathways. Cell 93:495-498.

Craven SE, El-Husseini AE, Bredt DS (1999) Synaptic targeting of the postsynaptic density protein PSD-95 mediated by lipid and protein motifs. Neuron 22:497-509.

Deguchi M, Hata Y, Takeuchi M, Ide N, Hirao K, Yao I, Irie M, Toyoda A, Takai Y (1998) BEGAIN (brain-enriched guanylate kinaseassociated protein), a novel neuronal PSD-95/SAP90-binding protein. J Biol Chem 273:26269-26272.

Doyle DA, Lee A, Lewis J, Kim E, Sheng M, MacKinnon R (1996) Crystal structures of a complexed and peptide-free membrane proteinbinding domain: molecular basis of peptide recognition by PDZ. Cell 85:1067-1076.

El-Husseini AE, Craven SE, Chetkovich DM, Firestein BL, Schnell E, Aoki C, Bredt DS (2000) Dual palmitoylation of PSD-95 mediates its vesiculotubular sorting, postsynaptic targeting, and ion channel clustering. J Cell Biol 148:159-172.

Garcia EP, Mehta S, Blair LA, Wells DG, Shang J, Fukushima T, Fallon JR, Garner CC, Marshall J (1998) SAP90 binds and clusters kainate receptors causing incomplete desensitization. Neuron 21:727-739.

Hillier BJ, Christopherson KS, Prehoda KE, Bredt DS, Lim WA (1999) Unexpected modes of PDZ domain scaffolding revealed by structure of nNOS-syntrophin complex. Science 284:812-815.

Hsueh YP, Sheng M (1999) Requirement of N-terminal cysteines of PSD-95 for PSD-95 multimerization and ternary complex formation, but not for binding to potassium channel Kv1.4. J Biol Chem 274:532-536.

Hsueh YP, Kim E, Sheng M (1997) Disulfide-linked head-to-head multimerization in the mechanism of ion channel clustering by PSD-95. Neuron 18:803-814.

Kennedy MB (1998) Signal transduction molecules at the glutamatergic postsynaptic membrane. Brain Res Brain Res Rev 26:243-257.

Kim E, Sheng M (1996) Differential K+ channel clustering activity of PSD-95 and SAP97, two related membrane-associated putative guanylate kinases. Neuropharmacology 35:993-1000.

Kim E, Niethammer M, Rothschild A, Jan YN, Sheng M (1995) Clustering of Shaker-type $\mathrm{K}+$ channels by interaction with a family of membrane-associated guanylate kinases. Nature 378:85-88.

Kim E, Cho KO, Rothschild A, Sheng M (1996) Heteromultimerization and NMDA receptor-clustering activity of Chapsyn-110, a member of the PSD-95 family of proteins. Neuron 17:103-113.

Kim E, Naisbitt S, Hsueh YP, Rao A, Rothschild A, Craig AM, Sheng M (1997) GKAP, a novel synaptic protein that interacts with the guanylate kinase-like domain of the PSD-95/SAP90 family of channel clustering molecules. J Cell Biol 136:669-678.

Kim JH, Liao D, Lau LF, Huganir RL (1998) SynGAP: a synaptic
RasGAP that associates with the PSD-95/SAP90 protein family. Neuron 20:683-691.

Kornau HC, Schenker LT, Kennedy MB, Seeburg PH (1995) Domain interaction between NMDA receptor subunits and the postsynaptic density protein PSD-95. Science 269:1737-1740.

Kuhlendahl S, Spangenberg O, Konrad M, Kim E, Garner CC (1998) Functional analysis of the guanylate kinase-like domain in the synapseassociated protein SAP97. Eur J Biochem 252:305-313.

Lahey T, Gorczyca M, Jia XX, Budnik V (1994) The Drosophila tumor suppressor gene dlg is required for normal synaptic bouton structure. Neuron 13:823-835.

Lim S, Naisbitt S, Yoon J, Hwang JI, Suh PG, Sheng M, Kim E (1999) Characterization of the shank family of synaptic proteins. Multiple genes, alternative splicing, and differential expression in brain and development. J Biol Chem 274:29510-29518.

Masuko N, Makino K, Kuwahara H, Fukunaga K, Sudo T, Araki N, Yamamoto H, Yamada Y, Miyamoto E, Saya H (1999) Interaction of NE-dlg/SAP102, a neuronal and endocrine tissue-specific membraneassociated guanylate kinase protein, with calmodulin and PSD- 95/ SAP90. A possible regulatory role in molecular clustering at synaptic sites. J Biol Chem 274:5782-5790.

McGee AW, Bredt DS (1999) Identification of an intramolecular interaction between the SH3 and guanylate kinase domains of PSD-95. J Biol Chem 274:17431-17436.

Moarefi I, LaFevre-Bernt M, Sicheri F, Huse M, Lee CH, Kuriyan J, Miller WT (1997) Activation of the Src-family tyrosine kinase Hck by SH3 domain displacement. Nature 385:650-653.

Naisbitt S, Kim E, Weinberg RJ, Rao A, Yang FC, Craig AM, Sheng M (1997) Characterization of guanylate kinase-associated protein, a postsynaptic density protein at excitatory synapses that interacts directly with postsynaptic density-95/synapse-associated protein 90 . J Neurosci 17:5687-5696.

Naisbitt S, Kim E, Tu JC, Xiao B, Sala C, Valtschanoff J, Weinberg RJ, Worley PF, Sheng M (1999) Shank, a novel family of postsynaptic density proteins that binds to the NMDA receptor/PSD-95/GKAP complex and cortactin. Neuron 23:569-582.

O'Brien RJ, Lau LF, Huganir RL (1998) Molecular mechanisms of glutamate receptor clustering at excitatory synapses. Curr Opin Neurobiol 8:364-369.

Pawson T, Scott JD (1997) Signaling through scaffold, anchoring, and adaptor proteins. Science 278:2075-2080.

Sheng M, Wyszynski M (1997) Ion channel targeting in neurons. Bioessays 19:847-853.

Sheng M, Tsaur ML, Jan YN, Jan LY (1992) Subcellular segregation of two A-type K+ channel proteins in rat central neurons. Neuron 9:271-284.

Sicheri F, Moarefi I, Kuriyan J (1997) Crystal structure of the Src family tyrosine kinase Hck. Nature 385:602-609.

Takeuchi M, Hata Y, Hirao K, Toyoda A, Irie M, Takai Y (1997) SAPAPs. A family of PSD-95/SAP90-associated proteins localized at postsynaptic density. J Biol Chem 272:11943-11951.

Thomas U, Kim E, Kuhlendahl S, Koh YH, Gundelfinger ED, Sheng M, Garner CC, Budnik V (1997) Synaptic clustering of the cell adhesion molecule fasciclin II by discs- large and its role in the regulation of presynaptic structure. Neuron 19:787-799.

Tiffany AM, Manganas LN, Kim E, Hsueh YP, Sheng M, Trimmer JS (2000) PSD-95 and SAP97 exhibit distinct mechanisms for regulating $\mathrm{K}(+)$ channel surface expression and clustering. J Cell Biol 148:147-158.

Topinka JR, Bredt DS (1998) N-terminal palmitoylation of PSD-95 regulates association with cell membranes and interaction with $\mathrm{K}+$ channel Kv1.4. Neuron 20:125-134.

Tu JC, Xiao B, Naisbitt S, Yuan JP, Petralia RS, Brakeman P, Doan A, Aakalu VK, Lanahan AA, Sheng M, Worley PF (1999) Coupling of mGluR/Homer and PSD-95 complexes by the Shank family of postsynaptic density proteins. Neuron 23:583-592.

Woods DF, Bryant PJ (1991) The discs-large tumor suppressor gene of Drosophila encodes a guanylate kinase homolog localized at septate junctions. Cell 66:451-464.

Woods DF, Hough C, Peel D, Callaini G, Bryant PJ (1996) Dlg protein is required for junction structure, cell polarity, and proliferation control in Drosophila epithelia. J Cell Biol 134:1469-1482.

Xu W, Harrison SC, Eck MJ (1997) Three-dimensional structure of the tyrosine kinase c-Src. Nature 385:595-602.

Ziff EB (1997) Enlightening the postsynaptic density. Neuron 19:11631174. 